\title{
Virtual Reality in Education: Learning a Foreign Language
}

\author{
Darque Pinto $^{1(\otimes)}$, Bruno Peixoto ${ }^{1}$, Aliane Krassmann ${ }^{2}$, Miguel Melo ${ }^{3}$, \\ Luciana Cabral ${ }^{4,5}$, and Maximino Bessa ${ }^{1,3}$ \\ 1 Universidade de Trás-os-Montes e Alto Douro, Vila Real, Portugal \\ Darquepinto@gmail.com \\ 2 Universidade Federal do Rio Grande do Sul, Porto Alegre, Brazil \\ 3 INESC TEC, Porto, Portugal \\ 4 CITCEM, Porto, Portugal \\ 5 Instituto Politécnico de Bragança, Bragança, Portugal
}

\begin{abstract}
There are still open questions about the effectiveness of Virtual Reality (VR) in Education when compared to conventional learning methods. This paper studies the feasibility of a VR-based learning tool and the possible differences in knowledge retention across a VR learning method and a conventional audio method, when it comes to learning a foreign language. Also, the students' sense of presence and satisfaction were studied. For such purpose, a user study was conducted and results revealed that while presence and satisfaction were higher in Virtual Reality, the knowledge retention score remains the same across both experimental conditions.
\end{abstract}

Keywords: Virtual reality $\cdot$ Language learning $\cdot$ Media in education • Simulations

\section{Introduction}

With the widespread of social networks, virtual communities, 3D virtual worlds, and human avatars, education has been challenged to develop new didactic approaches and strategic learning methods, integrating technology to enhance learning performance and motivation. The attention of learners is no longer attracted to the same resources that were used in the past, it is necessary to innovate to make students feel engaged in the learning process.

Among the different technologies available, Virtual Reality (VR) has been proven to be valuable for educational purposes $[1,2]$. VR can be described as a technology that provides a sensation of being immersed in a digital environment [3]. The main objective of VR is to make the user feel close to another reality, using the human's five senses. This technology integrates a diversity of devices that may be used to help create a realistic and multisensory experience (e.g., Head-Mounted Displays (HMD), motion tracking, etc.) [4]. This enables us to replicate or to create 
different convincing realities and simulations, allowing to change the users' perception of the world and their capacity to store, share and transmit information, a fact that increased the interest of educational researchers.

As a person experiences the computer-generated environment rather than the actual physical location, the computer world becomes the user's world, which is called the sense of presence [5]. For Slater and Wilbur [6], the fundamental idea is that individuals with a high sense of presence experience the virtual environment as the reality more involving than the surrounding physical world, to the point of considering it as a place visited. That is why attention and involvement (or engagement) are common responses from users associated with the sense of presence [7].

In this sense, North and North [2] and Makransky, Terkildsena and Mayer [8] emphasize that sense of presence in VR can contribute to learning. This is because, according to Hassell et al. [9], when experiencing high levels of presence concentration, which is a key factor for learning, will be focused on the activity that occurs in the virtual environment.

Felix [10] explains that "skills and knowledge are best acquired within realistic contexts[...]" suggesting that learning can be enhanced by the stimulus of interaction with environments and people [11]. That is, placing the student in the real context where the knowledge is applied, which is called situated learning [12]. In the case, of foreign language, it is common to observe that people decide to study abroad to fast acquire listening and speaking skills, while inserted in its real context of the language existence, where they will literally be surrounded by it. Thus, it is highlighted the benefits of this pedagogic approach for foreign language learning.

Emerging VR technology offers an authentic hyper-immersive experience at an affordable price and in a technically feasible format for large cohort teaching for the first time. It is therefore absolutely relevant and convenient to investigate how VR might impact the student learning [13]. Thus, we hypothesize that (1) is it possible to use VR-based learning tools to learn a foreign language, and (2) that such VR-based learning tools are more effective regarding the learning process. Thus, we propose such a virtual environment and conduct a user study to clarify the tangible results of VR for education, focusing on the knowledge retention of listening exercises of foreign language learning, in comparison with the Audio format.

The remainder of the paper is structured as follows: we start reviewing articles from the same area, followed by a description of the used methods and their results. We finally our study by adding some discussions comparing our results with the literature knowledge answering the researches' objective.

\section{Related Work}

The study by Ijaz, Bodgdanovych and Trescak [14] compared the outcomes of unsupervised learning from a text document, an educational video, and a 3D virtual world, all with the same content of historical facts from the ancient city 
of Uruk. From the experiment, a better performance was observed in the virtual world group over the text and video groups. Because of that, they reached the conclusion that studying in the virtual world would result in better academic performance, although the text group was the fastest mode.

Makransky, Terkildsen and Mayer [8] is one of the few studies that actually investigates immersive VR, comparing the learning outcomes with a desktop display version of the same simulation, in the context of Science. They found out that the students felt more presence with the VR but they actually learned less, compared with the low-immersive version. On their perspective, the lower results are justified by the excitement of using VR for the first time, as well as the overwhelming aspect of the more cognitive load inherent with the use of HMD.

Christopoulos, Conrad and Shukla [15] conducted a research in which they identified that the interactivity of virtual objects, combined with the interactions between the peers, helped the students feel a sense of presence in the virtual world and, consequently, experience the learning material more intensively; something that, according to the authors, turned out to be more effective than "just studying".

It is reasonable to assume that with increased realism the student's engagement with a virtual environment will be improved and learning outcomes will as well outdo as a consequence. However, insufficient evidence is available in the existing literature to confirm those links [13].

In this research, it is intended to endorse the efficiency of Virtual Reality aiming the learning of a foreign language, compared with a conventional use. Our goal is to analyze the sense of presence, satisfaction, and knowledge retention between VR and audio.

\section{Methods}

\subsection{Participants and Design}

The experiment was performed by 12 participants, ages between 12 and 15 years old $(\mathrm{M}=13.67, \mathrm{SD}=0.745)$, attending classes of J5 level of English (being J0 the basic level and J8 the advanced), at a private English school in Portugal, therefore guaranteeing the sample consistency. Students were contacted and invited to participate in the experiment. Participation was entirely voluntary on the part of the students and there was no grading associated with the exercise. As volunteers were underage, written consent was provided by their legal guardians prior to the implementation of the study.

\section{$3.2 \quad$ Variables}

The independent variable was the learning mode (VR vs. Audio). The dependent variables were the sense of presence, satisfaction and knowledge retention, which were measured by a multiple-choice post-test questionnaire. 


\subsection{Materials}

The materials used in the study included a simulation delivered in two different versions (VR and Audio), a knowledge retention objective test, and a sense of presence and a satisfaction questionnaire. The knowledge retention test was written in English, and the questionnaires (presence and satisfaction) were written in the user's own language: Portuguese.

The simulation contains a dialogue similar to the ones commonly used in the English listening exercises. It was created by using the Unity game engine and it consisted of two scenarios. The first scenario present a formal English dialogue (in an office), while the second one presents an informal conversation (in a pub). The scenarios were filled with props to characterize it, and Non-Player Characters (NPCs) to meet the dialog script. In both scenarios, the user would be placed at a table surrounded by the NPCs engaged in a narrative fitting each scenario. The formal scenario takes 02:36 min while the informal takes 01:29 min. For the two modes of instruction, the simulation was the same, just varying the ways of the stimulus: Audio and VR.

Two laptop computers were used for the experiment: an ASUS ROG G752 (Intel Core i7-7700HQ processor and NVIDIA GeForce 1070 graphics card) for the VR experience, and an HP OMEN 17-an (Intel Core i7-7700HQ processor and NVIDIA GeForce 1050 graphics card) for the audio experience.

Concerning the visual VR stimulus, the HTC Vive HMD was used featuring a $110^{\circ}$ viewing angle and a resolution of $1080 \times 1200$ pixels per eye. The audio stimulus in VR was delivered with a noise cancellation headphones (Bose QuietComfort 25). The same headphones were used on the audio version. The VR stimulus was developed having into account the study by Melo et al. [16] to ensure that presence was not affected by the exposure time.

\subsection{Instruments}

Three instruments were used in this research, each one to evaluate on of the three aspects investigated: sense of presence, satisfaction and knowledge retention. To measure the sense of presence, the validated Portuguese version of Igroup Presence Questionnaire (IPQ) [17] was selected, because it gives insights values three dimensions: spatial presence, involvement, and experienced realism. It contains fourteen likert scale affirmations, where the users answered between one (totally disagree) and five (totally agree).

The After-Scenario Questionnaire (ASQ) [18] was used to evaluate the satisfaction of the participant with the system usability, namely the following: ease of task completion, time to complete and adequacy of support information. It is composed with six likert scale affirmations where the users answered between one (totally disagree) and seven (totally agree).

An eight questions multiple-choice test was created by the English teachers of the school, based on the story that is presented in both scenarios, thought of to evaluate students' knowledge retention after the experiment. Since the test has a full value of $100 \%$, each answer has a value of $12.5 \%$. The questions of this test are presented below. 
Questions for the formal scenario:

1. Why is Paul Jones not at the meeting? Because he went to:

2. What was the Director worried about?

3. When was the meeting held?

4. What was the main topic of the discussion at the meeting?

Questions for the informal scenario:

5. At the pub, how are they feeling at first?

6. Was Anna able to contact her colleague who was abroad?

7. Which of the countries listed below did Paul not go to?

8. Were the colleagues happy at the end of the meeting at the pub?

The instruments were answered in a pen-and-paper based format, immediately after the student undertook the experiment, in order to ensure high response rates and the currency of responses.

\subsection{Procedure}

Participants were tested individually in a room, with a controlled environment, at the English school. A sociodemographic questionnaire was given before the beginning of the experience. To avoid confusing the results with a specific type of content, the two different modes of instruction (audio and VR) were tested for both the scenarios, in a random order. Each participant first tested one scenario in one mode and the other using the other mode of instruction, alternatively. In this way, after each scenario, the user had to answer a knowledge test, a presence, and a satisfaction questionnaire.

Prior to the experiment itself, participants in the VR condition entered in a training room for one minute, which was the same scenario of the simulation, but without the NPCs, just the scene. The aim was to allow the subjects, who could not be familiar with virtual environments, to become accustomed to maneuvering the HMD. Overall, each participation took around $20 \mathrm{~min}$, including the instruments filling.

\section{Results}

Regarding the presence and satisfaction, VR condition achieved better results than audio. In Fig. 1 we have a graph that shows the results of the questionnaires and in Table 1 we have the same more detailed results.

In Fig. 2 it is represented the percentage of the knowledge retention test, separated by scenario, being $100 \%$ all the questions answered correctly and $0 \%$ all wrong answers. In none of the conditions it was reached the minimum to consider as a positive result (above 50\%). Apart from this low outcome, some differences were observable between both scenarios. In the formal scene, virtual reality had $30 \%$ of correct answers and the audio got a better result with more than $30 \%$. In the informal scene, we observed the opposite. As regards, the final results virtual reality had more than $40 \%$ and audio less than that value. 


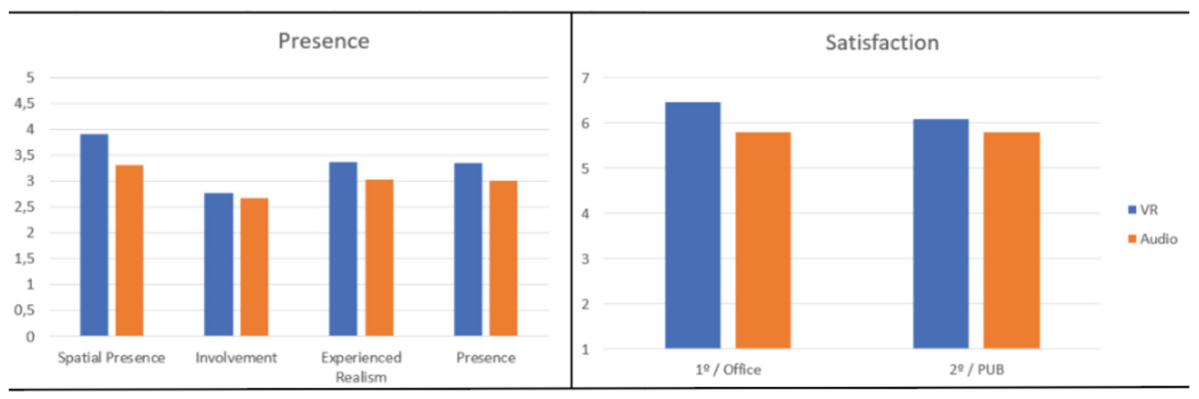

Fig. 1. IPQ and ASQ graph

Table 1. M and S.D Table

\begin{tabular}{|c|c|c|c|c|}
\hline & \multicolumn{4}{|c|}{ Experiment } \\
\hline & \multicolumn{2}{|c|}{ VR } & \multicolumn{2}{|c|}{ Audio } \\
\hline Presence Scale & M & S.D. & $\mathbf{M}$ & S.D. \\
\hline \multirow{6}{*}{$\begin{array}{c}\text { Spatial Presence } \\
\text { Involvement } \\
\text { Experienced Realism } \\
\text { Presence }\end{array}$} & 3.907 & .394 & 3.310 & .412 \\
\hline & 2.778 & .845 & 2.679 & 1.083 \\
\hline & 3.361 & .458 & 3.036 & .891 \\
\hline & 3.349 & .362 & 3.008 & .579 \\
\hline & \multicolumn{4}{|c|}{ Experiment } \\
\hline & \multicolumn{2}{|c|}{ VR } & \multicolumn{2}{|c|}{ Audio } \\
\hline Satisfaction Scale & M & S.D. & M & S.D. \\
\hline Satisfaction & 6.3 & .48 & 5.8 & .77 \\
\hline
\end{tabular}

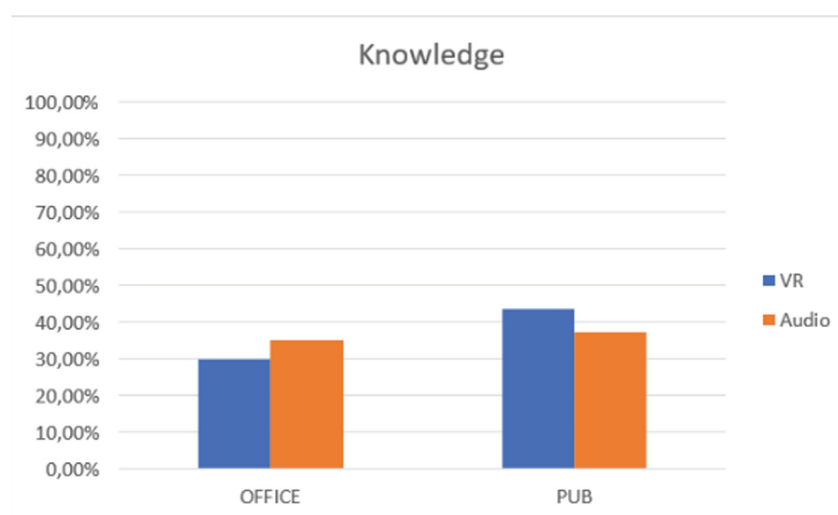

Fig. 2. Knowledge test graph

\section{Discussion}

After reviewing the knowledge retention test and the IPQ and ASQ questionnaires answered by the participants, some interesting results were collected. 
In terms of the sense of presence, we could have expected that Virtual Reality would have better results than audio and in Fig. 1 and Table 1 we can see the difference between them happening on the values. Thus, our results corroborate the Makransky, Terkildsen, and Mayer [8] study where they found out that the presence is bigger in VR condition when comparing learning between VR and a desktop display, when testing the learning of science.

The same contrast happened with the measure of satisfaction. VR is a preference among participants. We theorize that this happens due to the fact that it was the very first time trying Virtual Reality for the most of them, causing therefore enthusiasm and worship for this kind of learning.

In Fig. 2 we can also see that the results of the knowledge retention test are lower than we could expect but they are very similar with the audio version.

Since VR was better than the conventional use in one of both scenario, and vice versa, the results about knowledge retention were inconclusive. Thus, we could not corroborate the Ijaz, Bodgdanovych, and Trescak [14] study, where they reached the conclusion that VR was more efficient for a better academic performance, comparing to a conventional method (text version, in their case). Regarding this difference between results, we speculate that this happens due to the same fact as the satisfaction results, it was the first time experimenting VR for most of the students, which can lead to some natural distraction. While some of the participants were enjoying the environment itself, they did not pay attention to the dialogue. This cannot justify the difference between scenarios and that is why the knowledge retention results are inconclusive.

Since, according to Christopoulos, Conrad, and Shukla [15] study, interactivity with virtual objects make the participants learn more effectively than just "studying", this kind of tool in VR could well be a solution for better results in the knowledge retention. This conclusion may well lead to another investigation including interactivity in the scenarios, comparing results with and without it. However, at this stage, we aimed comparing VR with "listening". The mentioned interaction is thought of to be a huge advantage, comparing to audio alone.

\section{Conclusions}

According to our investigation, we can conclude that the VR results of the knowledge retention test are very similar to the audio version, validating our hypothesize (1) where we state that it is possible to use VR-based learning tools to learn a foreign language.

However, also because the learning outcomes were very similar in both conditions, the results are inconclusive for the assumption (2) that VR-based learning tools are more effective regarding the learning process.

The investigation of the sense of presence and the satisfaction aspects increased the analysis, showing that students actually felt more present and satisfied in the VR condition, which points out to the potential benefits of integrating this technology in teaching, to improve the students' positive feelings, besides capturing and maintaining their attention, especially in disciplines considered more "boring". Slater [19] have demonstrated that users whose primary 
representation systems are visual are more likely to experience presence in a visual virtual environment than users whose primary representation systems are auditory or kinaesthetic. This claims for further investigation on whether students learning preferences (learning styles) could influence or not in the outcomes of the treatments provided in this research.

Within the limitations of the study, it is certainly the novelty factor of VR technology, which may have influenced the learning outcomes. Nevertheless, it is expected that VR will become more popular as the devices become cheaper, so it is necessary to study its impact on education to be prepared for this integration. As a new medium, VR offers something that other media used in education cannot, that is, an immersive experience that can place the student in a specific learning context, evoking similar reactions and emotions [2]. Thus, the potential of situated learning [12] for foreign language must be explored, which can be a good cost-effective approach.

In addition, the small sample size is clearly a limitation of the study, avoiding to extrapolate the results to other contexts. Also, the age group of the subjects may have distort the measure of the sense of presence, because as they were under age they probably do not have the experience of being in an office or in a pub (formal and informal scenarios, respectively), and the instrument had assertions as "the experience in the virtual environment seemed to me as real as my daily experiences" and "the virtual environment seemed to me as real as the world I know" [7]. Thus, future endeavours are planned to extend this study to more participants, in a context of adults and, if possible, with subjects familiar with VR technology to mitigate the novelty effects.

In conclusion, this research makes a contribution to understanding the potential of VR technology to support and enhance situated learning, elucidating the main advantages and drawbacks in the context of listening exercises for foreign language learning.

Acknowledgments. This work is financed by the ERDF - European Regional Development Fund through the Operational Programme for Competitiveness and Internationalisation - COMPETE 2020 Programme and by National Funds through the Portuguese funding agency, FCT - Fundação para a Ciência e a Tecnologia within project POCI-01-0145-FEDER-028618 entitled PERFECT - Perceptual Equivalence in virtual Reality For authEntiC Training.

We want to thank Marta Peixoto, Claúdia Peixoto and the others teachers from the English Institute of Vila Real for their support and collaboration in this study.

\section{References}

1. Mikropoulos, T.: The unique features of educational virtual environments. In: Proceedings E-society 2006, International Association for Development of the Information Society, vol. 1 (2006)

2. North, M., North, S.: The sense of presence exploration in virtual reality therapy. J. Univers. Comput. Sci. 24, 72-84 (2018) 
3. Góomez-García, M., Trujillo-Torres, J.M., Aznar-Díaz, I., Cáceres-Reche, M.P.: Augment reality and virtual reality for the improvement of spatial competences in physical education (2018)

4. Chesham, R.K., Malouff, J.M., Schutte, N.S.: Meta-analysis of the efficacy of virtual reality exposure therapy for social anxiety. Behav. Chang. 35(3), 152-166 (2018). https://doi.org/10.1017/bec.2018.15

5. Witmer, B.G., Singer, M.J.: Measuring presence in virtual environments: a presence questionnaire. Presence Teleoperators Virtual Environ. 7. https://doi.org/10.1162/ 105474698565686.

6. Slater, M., Wilbur, S.: A framework for immersive virtual environments (FIVE): speculations on the role of presence in virtual environments. Presence Teleoperators Virtual Environ. 6, 603 (1997). https://doi.org/10.1162/pres.1997.6.6.603

7. Lessiter, J., Freeman, J., Keogh, E., Davidoff, J.: A cross-media presence questionnaire: the ITC-sense of presence inventory. Presence 10, 282-297 (2001). https:// doi.org/10.1162/105474601300343612

8. Makransky, G., Terkildsen, T.S., Mayer, R.E.: Adding immersive virtual reality to a science lab simulation causes more presence but less learning. Learn. Instr. https://doi.org/10.1016/j.learninstruc.2017.12.007

9. Hassell, K., Coutin, P., Nugegoda, D.: A novel approach to controlling dissolved oxygen levels in laboratory experiments. J. Exp. Mar. Biol. Ecol. 371, 147-154 (2009). https://doi.org/10.1016/j.jembe.2009.01.013

10. Felix, U.: The web as a vehicle for constructivist approaches in language teaching. ReCALL 14(1), 2-15 (2002). https://doi.org/10.1017/S0958344002000216

11. Yeh, Y.-L., Lan, Y.-J., Lin, Y.-T.R.: Gender-related differences in collaborative learning in a $3 \mathrm{D}$ virtual reality environment by elementary school students. J. Educ. Technol. Soc. 21(4), 204-216 (2018)

12. Matusov, E., Bell, N., Rogoff, B.: Situated learning: legitimate peripheral participation. Am. Ethnol. 21, 918-919 (1994). https://doi.org/10.1525/ae.1994.21.4. 02a00340. jean lave, etienne wenger

13. Wang, R., Newton, S., Lowe, R.: Experiential learning styles in the age of a virtual surrogate. Int. J. Arch. Res. ArchNet-IJAR 9, 93-110 (2015). https://doi.org/10. 26687/archnet-ijar.v9i3.715

14. Ijaz, K., Bogdanovych, A., Trescak, T.: Virtual worlds vs books and videos in history education. Interact. Learn. Environ. 25(7), 904-929 (2017). https://doi. org/10.1080/10494820.2016.1225099

15. Christopoulos, A., Conrad, M., Shukla, M.: Interaction with educational games in hybrid virtual worlds. J. Educ. Technol. Syst. 46(4), 385-413 (2018). https://doi. org $/ 10.1177 / 0047239518757986$

16. Melo, M., Vasconcelos-Raposo, J., Bessa, M.: Presence and cybersickness in immersive content: effects of content type, exposure time and gender. Comput. Graph. 71, 159-165 (2018). https://doi.org/10.1016/j.cag.2017.11.007

17. Schubert, T., Friedmann, F., Regenbrecht, H.: The experience of presence: factor analytic insights. Presence Teleoperators Virtual Environ. 10(3), 266-281 (2001). https://doi.org/10.1162/105474601300343603

18. Lewis, J.R.: IBM computer usability satisfaction questionnaires: psychometric evaluation and instructions for use. Int. J. Hum. Comput. Interact. 7(1), 57-78 (1995). https://doi.org/10.1080/10447319509526110

19. Slater, M., Steed, A., McCarthy, J., Maringelli, F.: The influence of body movement on subjective presence in virtual environments. Hum. Factors 40, 469-477 (1998). https://doi.org/10.1518/001872098779591368 